Please do not remove this page

RMIT

UNIVERSITY

\title{
Noise estimation in spherical coordinates for color image restoration
}

Hore, E; Qui, Bin; Wu, Henry

https://researchrepository.rmit.edu.au/esploro/outputs/9921858523501341/filesAndLinks?institution=61RMIT_INST\&index=null

Hore, E., Qui, B., \& Wu, H. (2005). Noise estimation in spherical coordinates for color image restoration. Optical Engineering, 44(4), 21-29. https://doi.org/10.1117/1.1883696

Document Version: Published Version

Published Version: https://doi.org/10.1117/1.1883696

Repository homepage: https://researchrepository.rmit.edu.au

(c) Copyright (2005) Society of Photo-Optical Instrumentation Engineers. One print or electronic copy may be made for personal use only. Systematic reproduction and distribution, duplication of any material in this paper for a fee or for commercial purposes, or modification of the content of the paper are prohibited. Society of Photo-Optical Instrumentation Engineers

Downloaded On 2023/04/26 18:07:25 +1000 


\section{Noise estimation in spherical coordinates for color image restoration}

\author{
E. S. Hore \\ B. Qiu \\ H. R. Wu \\ Monash University \\ School of Computer Science and Software \\ Engineering \\ P.O. Box 26 \\ Victoria 3800, Australia \\ E-mail: ehore@csse.monash.edu.au
}

\begin{abstract}
A new adaptive filter is presented, designed for the restoration of color images corrupted with an arbitrary noise model. The proposed filter is based on order-statistics restoration techniques and performs a linear combination of a number of difference measures to estimate the noise present in each pixel. To reduce color artifacts, the filtering is performed in a color space that decouples the intensity and color information. The choice of which color space is arbitrary; however, from experimentation it has been found that a spherical coordinate representation of the RGB pixel vectors provides the best results. As the results show, the proposed filter outperforms the current state of the art techniques for impulse, additive, and multiplicative noise types. In addition to the proposed filter, a new color performance measure is introduced based on the mean square error; however, it can be calculated using any error measure that employs differences between two input signals. (1) 2005 Society of Photo-Optical Instrumentation Engineers. [DOI: 10.1117/1.1883696]
\end{abstract}

Subject terms: adaptive filter; color image restoration; order statistics; noise estimation.

Paper 030591 received Nov. 25, 2003; revised manuscript received Nov. 1, 2004; accepted for publication Nov. 12, 2004; published online Apr. 8, 2005.

\section{Introduction}

It is well known that nonlinear image restoration techniques outperform their linear counterparts in terms of reducing smoothing artifacts. ${ }^{1}$

A good review of the many varieties and classes of nonlinear filters can be found in Ref. 2, where it was shown that order-statistic filters provide excellent robustness to estimated filter parameters and performance for varying image characteristics. ${ }^{3,4}$ In addition, the operation of the basic order-statistics filter allows for both extendibility and compatibility with new and existing algorithms.

Possibly the most well-known order-statistics filter is the median filter. First introduced by Tukey ${ }^{5}$ for time-series analysis, it quickly became a favorite among the image processing research community. This quick acceptance of the median filter was due primarily to its simplicity, both conceptually and in terms of computation, as well as its robustness and good performance. As such, the standard median filter operation has formed the basis for many sophisticated order-statistics filters.

Another very successful order-statistics filter is the $L$ filter. ${ }^{6}$ The $L$ filter is derived from the set of robust estimators known as $L$ estimators and is essentially a linear combination of order statistics. By selecting appropriate coefficients, the $L$ filter can be generalized to act as a median, midpoint, $r$-ranked, or a trimmed mean filter. ${ }^{7,8}$ An extension to the $L$-filter technique is the weighted sum, or linear combination, filter operation, where a number of subfilter outputs are linearly combined. ${ }^{9,10}$

The majority of the $L$-filter processing operations such as coefficient optimization and the general form of the

0091-3286/2005/\$22.00 @ 2005 SPIE equations can be applied to the weighted sum filter as well. For the purpose of this work, the notation $W S$ filter will be used to denote the weighted sum filter to differentiate it from the standard $L$ filter.

The coefficients are specified to satisfy the location invariance constraint such that the sum of the elements in the coefficient set is equal to unity. If we let $C$ denote the coefficient set, i.e., $C=\left[c_{1}, c_{2}, \ldots, c_{L}\right]$, and $L$ the length of the set, then Eq. (1) illustrates the invariance constraint.

$\sum_{j=1}^{L} c_{j}=1$.

As pointed out in Refs. 6 and 11, when the constraint is applied it can be viewed as an unbiased estimate for the case of a constant signal corrupted with additive noise.

If we let $X$ denote the input pixel set and $L$ the length of the set, such that:

$X=\left[x_{1}, x_{2}, \ldots, x_{L}\right]$,

then the ordered input set, denoted as $\tilde{X}$, is specified as:

$\tilde{X}=\left[\tilde{x}_{(1)}, \tilde{x}_{(2)}, \ldots, \tilde{x}_{(L)}\right]$,

where $\tilde{x}_{(1)} \leqslant \tilde{x}_{(2)} \leqslant \ldots \leqslant \tilde{x}_{(L)}$.

The basic form of an $L$ filter is given in Eq. (4). Note that it is identical to a linear filter, except for the fact that the input set has been ordered.

$Y=\sum_{i=1}^{L} c_{i} \tilde{x}_{i}$ 
In the case of the WS filters, Eq. (4) has the same form, except that $\tilde{X}$ is replaced by the set of subfilter outputs. Note that in addition to the change in $\tilde{X}$, then the length of the set being combined is not restricted to $L$, as in Eq. (4), but rather depends on the number of subfilters employed.

In Ref. 12 , an adaptive $L$-filter technique was proposed using the least mean square (LMS) algorithm for image restoration. The optimization of the coefficients was carried out using the original image as a reference, and although this introduces dependencies on the training set, the adaptive LMS $L$ filters are able to provide improved results.

It has been shown that a recursive implementation of the LMS $L$ filter provides a further improvement over the nonrecursive algorithm. ${ }^{13}$ As such, the recursive LMS optimization algorithm is employed by the proposed filter for training of the filter coefficients.

\subsection{Color Image Restoration}

Recently, there have been a number of proposed color image restoration filters that use new and extend existing techniques. ${ }^{14-18}$ However, while these new multichannel filters provide promising results, they are generally designed to restore a particular attribute of color image pixels, i.e., pixel intensity or color.

Color image restoration using multichannel scalar orderstatistics filters can introduce color artifacts due to chromaticity shifts. Order-statistics techniques, particularly median-based filters, cause errors that arise from edge jitter. $^{19-21}$ Edge jitter occurs when the filter window lies over an edge in the underlying image structure. At the point where the center pixel first belongs to the edge, the median filter will replace the edge pixel with one of its nonedge neighbors. The actual amount of edge jitter is determined by both the thickness and orientation of the edge. ${ }^{21}$ In fact, the median filter will replace any center pixel that belongs to a segment in the image structure with less than $L / 2$ pixels under the filter window. For color images, the edge jitter results in both intensity and chromaticity shifts.

The proposed filter addresses false color artifacts by processing the input image in a color space that decouples the intensity and color information. For an RGB vector, the magnitude, which determines the intensity, is represented by a single scalar value. However, the direction, which defines the color, requires all three RGB components. In color spaces that decouple the intensity and color, at least two components are required to represent the color. Examples of such color spaces include $\mathrm{YC}_{b} \mathrm{C}_{r}, \mathrm{Lu}^{*} \mathrm{~V}^{*}$, and $\mathrm{HSV}$.

After performing a large number of experiments, it has been found that a simple spherical coordinate representation of the RGB pixel vectors can provide improved performance over many other color models. The details of the color space transformations are not given here; however, for more information, the reader is referred to Ref. 22.

The RGB color space is a Cartesian coordinate representation of a pixel in $R^{3}$ space. Each axis is orthogonal and linearly independent of the other, representing one of the three color primaries, red, green, and blue. As such, an RGB pixel vector is conceptually easy to visualize; it is simply a point in $R^{3}$ space. However, because the color and intensity information is tightly coupled, it can be difficult to determine the perceived color and intensity of a pixel from the RGB components. Essentially, the intensity is the distance between the pixel vector and the origin, while the color is the direction of the vector.

The coupling of the intensity and color information, or magnitude and direction, also cause difficulties when Cartesian coordinates are used in other science disciplines. For example, spherical coordinates are commonly used when the problem at hand may be to ascertain the angular velocity of an object in $R^{3}$ space.

A spherical coordinate representation has many advantages, including a relatively fast computation time, as well as being easy to visualize and relate back to the original vector in Cartesian space. In addition, spherical coordinates decouple the intensity and color information of the pixel vector. As is shown in Sec. 3, a spherical coordinate representation of the RGB pixel vectors provides improved results when compared to other color spaces that also decouple the intensity and color attributes.

\subsection{Noise Models}

The three noise models that are used throughout this work are impulse noise, additive noise, and multiplicative noise. For additive and multiplicative noise models, all pixels are potentially corrupt with the degradation models defined per Eqs. (5) and (6), respectively.

$\hat{I}_{(s, t)}=I_{(s, t)}+\epsilon_{(s, t)}$.

$\hat{I}_{(s, t)}=I_{(s, t)} \times \epsilon_{(s, t)}$.

In Eqs. (5) and (6), $\hat{I}$ and $I$ are the corrupt and original images, respectively. $W$ and $H$ are the width and height of the images, while the subscripts $s$ and $t$ denote the image coordinates such that $1 \leqslant s \leqslant W$ and $1 \leqslant t \leqslant H$. The noise introduced to each pixel, denoted here as $\epsilon_{(s, t)}$, is a random value drawn from an arbitrary distribution.

Note, however, that the distribution for each is not related to the degradation model as such. For example, the additive model can use either a Gaussian or uniform distribution. The noise parameters specify the distribution properties, and hence, the characteristics of $\epsilon$. In this work, the zero-mean Gaussian and uniform distributions have been used. Equations (7) and (8) show the zero-mean Gaussian and uniform distribution models.

$P[\epsilon(i)]=\frac{1}{\sigma \sqrt{2 \pi}} \exp \left[-\frac{(x)^{2}}{2 \sigma^{2}}\right]$

In Eq. (7), $\sigma$ denotes the standard deviation of the distribution.

$P\left[\epsilon_{(s, t)}\right]=\left\{\begin{array}{ll}\frac{1}{b-a} & \text { for } a \leqslant x \leqslant b \\ 0 & \text { otherwise }\end{array}\right.$.

In Eq. (8), $a$ and $b$ denote the maximum and minimum of the distribution dynamic range. 
The distribution for impulse noise is uniform, where $a$ and $b$ are set to the maximum and minimum of the pixel value range. The expression given in Eq. (9) illustrates the impulse noise degradation model.

$\hat{I}_{(s, t)}=\left\{\begin{array}{l}I_{(s, t)} \text { with probability } 1-p \\ \epsilon_{(s, t)} \text { with probability } p\end{array}\right.$.

Here, $p$ is the probability of a corrupt pixel occurring, i.e., the noise density. The value of $\epsilon_{(s, t)}$ is a random value drawn from a uniform distribution and has a range equal to that of the pixel value range.

From Eqs. (5) and (6), it can be assumed that all of the image pixels will contain noise to some degree. Thus, using one of the input pixels as the output (as the median filter will) allows the noise in the selected pixel to pass through unattenuated. For color images, the noise will appear as an error in the intensity and/or color attributes of a pixel. For instance, the output pixel vector may be relatively close to the original in terms of its intensity, but still contain a significant error in terms of color, or vice-versa. In the worst case, both the intensity and color attributes of the output pixel vector will contain significant errors.

In the next section the details for the proposed filter are given. This is then followed by the experimental results, and finally the conclusions and possible future work.

\section{Proposed Filter}

The filter proposed in this work differs from many other order-statistics filters in that it uses the WS-filter technique to model noise characteristics. The noise is assumed to be of the type described in Sec. 1.2, specifically impulse noise, additive noise, and/or multiplicative noise. Note, however, that the user is not required to supply any a priori assumptions as to the type of noise that may be present in the image to be restored.

The WS filter estimates the amount of noise present in the center pixel using a number of variables that consist of difference measures. These measures are calculated as the difference between the center pixel and order-statistic subfilter outputs, as well as between the ordered input pixel values.

As previously mentioned, for color images the RGB pixel vectors are first transformed into spherical coordinates. The spherical coordinate representation of an RGB pixel has the form $\mathbf{x}=\left[x_{\rho}, x_{\theta}, x_{\phi}\right]$. The first component $x_{\rho}$ is the magnitude, or intensity, of the RGB vector, while the second and third components $x_{\theta}$ and $x_{\phi}$ represent the color. The element $x_{\theta}$ is the azimuth angle, measured between the $\mathrm{R}$ axis and the RGB vector projected onto the plane formed by the RG axis. The element $x_{\phi}$ is the elevation angle measured between the RGB vector and the RG plane. Equations (10), (11), and (12) give the transformations for $x_{\rho}, x_{\theta}$, and $x_{\phi}$, respectively, with $x_{r}, x_{g}$, and $x_{b}$ representing the RGB pixel vector components.

$x_{\rho}=\left(x_{r}^{2}+x_{g}^{2}+x_{b}^{2}\right)^{1 / 2}$,

$x_{\theta}=\tan ^{-1}\left(\frac{x_{g}}{x_{r}}\right)$,

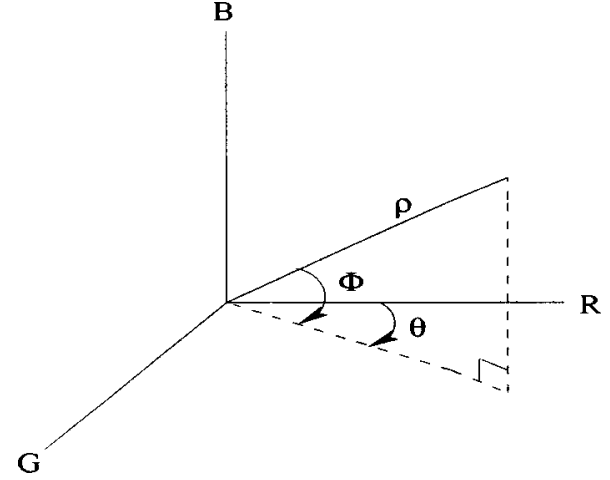

Fig. 1 Spherical coordinates.

$x_{\phi}=\tan ^{-1}\left[\frac{x_{b}}{\left(x_{r}^{2}+x_{g}^{2}\right)^{1 / 2}}\right]$.

Figure 1 illustrates the RGB spherical coordinates representation.

\subsection{Approximate and Combined Pixel Sets}

To improve the performance of the proposed filter, an iterative approach is employed, whereby an approximate image is generated using a preprocessing filter operation. For the preprocessing filter, it is advantageous to use a basic filter that has well-known characteristics and a low computation time. This allows the noise estimation and final output to be more computationally expensive while still maintaining the ability to operate in real-time applications.

It is also important that the preprocessing filter does not require optimized parameters such as coefficients or threshold levels. If the preprocessing filter behavior is determined by optimized parameters, then it will be dependent on the training set used and is likely to have highly nonlinear characteristics. This can make it extremely difficult to define the filter behavior analytically, and hence, there can be no $a$ priori knowledge about the artifacts that it may introduce into the approximate image.

During the filter operation, a total of three pixel sets are used, namely, the input pixel set, the approximate pixel set, and a combined pixel set. All three sets have the same length as the filter window. The elements of the input set, denoted as $X_{\epsilon}$, correspond to the pixels in the noisy input image that lie under the filter window. The approximate pixel set, denoted as $X_{a}$, is generated by switching the image under the filter window from the noisy input image to the approximate image. Thus, the elements in $X_{a}$ will be the pixels from the approximate image that have the same coordinates as the pixels in $X_{\epsilon}$.

The combined set, which is denoted as $X_{c}$, contains elements the are a combination of both $X_{\epsilon}$ and $X_{a}$. The center pixel of $X_{c}$ is drawn from the noisy input set, while the center pixel neighbors are taken from the approximate pixel set $X_{a}$. Hence, $X_{c}$ has the same center pixel as $X_{\epsilon}$ and the same center pixel neighbors as $X_{a}$. The following expression illustrates the three pixel sets.

$X_{a}=\left\{x_{a_{1}}, \ldots, x_{a_{c p}}, \ldots, x_{a_{L}}\right\}$, 
$X_{\epsilon}=\left\{x_{\epsilon_{1}}, \ldots, x_{\epsilon_{c p}}, \ldots, x_{\epsilon_{L}}\right\}$

$X_{c}=\left\{x_{a_{1}}, \ldots, x_{\epsilon_{c p}}, \ldots, x_{a_{L}}\right\}$.

Here, $c p=(L+1) / 2$ is the index of the center pixel and the elements $x_{a_{i}}$ and $x_{\epsilon_{i}}$ denote pixels from the approximate and noisy images, respectively.

Because the filter operation is performed on each pixel channel individually, the ordering technique can be a standard marginal ordering operation based on the raw scalar values. However, by using a reduced aggregate ordering, not unlike that of the vector median filters, ${ }^{19}$ information can be gathered as to how the scalar components of each set relate to the local image structure. In addition, the reduced aggregate ordering technique also simplifies the process of outlier detection, as the pixel components are ordered according to their aggregate interpixel distance. For additive and multiplicative noise, where the amount of noise in the center pixel may not cause it to appear as an outlier, this information can be used successfully to detect discontinuities in the image structure.

\subsection{WS-Filter Noise Model}

The amount of the noise in a pixel component, denoted as $\epsilon$, is estimated using a $W S$-filter noise model. The noise model has the standard form shown in Eq. (14).

$\hat{\boldsymbol{\epsilon}}=c_{0}+\sum_{i=1}^{M} v_{i} c_{i}$.

In Eq. (14), $\hat{\epsilon}$ is the estimate of $\epsilon, M$ is the number of independent $W S$-filter variables ( $M=3$ in this case), $v_{i}$ is the $i$ 'th independent variable and $c_{i}$ is the $i$ 'th coefficient. The constant coefficient $c_{0}$ is equivalent to the intercept on the $y$ axis of the linear function. The reader will note that most general $L$ and $W S$ filters do not use the constant coefficient $c_{0}$. Including $c_{0}$ gives the $W S$ filter the same form as a multiple linear regression model and has been found to provide greater performance and robustness.

The model consists of three independent variables. The first variable is the signed difference between the noisy center pixel and the approximate pixel at the same location, as defined in Eq. (15):

$v_{1}=X_{\epsilon(c p)}-X_{a(c p)}$,

where $X_{\epsilon(c p)}$ is the center pixel from the noisy set and $X_{a(c p)}$ is the center pixel from the approximate pixel set.

This is essentially the difference between the center pixel and the mean or median of the filter window, depending on which filter was used to generate the approximate image. The measure provides an initial, albeit rough, estimate of the distance between the center pixel and the original pixel value. Hence, it provides some indication as to the value of $\epsilon$ in the center pixel.

The second independent variable is the distance between the center pixel and one of its two nearest neighbors in the ordered combined set. This measure can provide a more detailed indication of how the center pixel relates to the local signal distribution. Because the filter window is square with odd dimensions, if the center pixel component belongs to an edge or fine detail segment, then at least one of its neighboring pixels will also belong to the same contour segment. In such circumstances, they can provide a more accurate estimate of the noise in the center pixel than the first initial estimate recorded in the first independent variable $v_{1}$.

In the case where the center pixel does not belong to an edge, however, the first variable combined with the two nearest-neighbor distance will still provide a more accurate estimate than either of these measures used by themselves. Similarly, if the center pixel component does not belong to an edge, or if no edge is present under the filter window, then at least one of the two nearest neighbors in the ordered combined pixel set $\tilde{X}_{c}$ will still belong to the same nonedge segment.

The indices of the two nearest neighbors can be obtained using the following expression.

$\{i a, i b\}=\left\{\begin{array}{ll}c p-1, c p+1 & \text { for } 1<c p<L \\ L-1, L-2 & \text { for } c p=L \\ 2,3 & \text { for } c p=1\end{array}\right.$.

Here, $i a$ and $i b$ denote the indices of the two neighbors and $c p$ is again the center pixel index.

Once the center pixel nearest-neighbor indices have been obtained, the distances are calculated as a signed scalar difference, shown in Eq. (17).

$v_{2_{a}}=\tilde{x}_{c(c p)}-\tilde{x}_{c(i a)}$,

$v_{2_{b}}=\tilde{x}_{c(c p)}-\tilde{x}_{c(i b)}$.

Here, $\tilde{x}_{c(c p)}$ is the center pixel component from the ordered combined set and $\tilde{x}_{c(i a)}, \tilde{x}_{c(i b)}$ are the two nearest neighbors at locations $i a$ and $i b$, respectively. The final value for the second variable, denoted as $v_{2}$, is then selected from either $v_{2}$ or $v_{2_{b}}$ in the following manner.

The instances where $v_{2_{a}}$ or $v_{2_{b}}$ may become inaccurate is generally when the center pixel is placed at the high end of the ordered combined set. In this situation, there is the possibility that either the center pixel component and its nearest-neighbor component, i.e., $\min \left(v_{2_{a}, v_{2}}\right)$, are both highly corrupt, or that they belong to a small segment in the image structure. The task, therefore, is one of determining whether or not the center pixel and its nearest neighbor belong to the image structure or whether they are simply a cluster of highly corrupt pixel components.

This is achieved by temporarily increasing the filter window dimensions by an arbitrary amount. For the work presented here, an increase of 4 has been used, such that a $3 \times 3$ window would be increased to $7 \times 7$, or a $5 \times 5$ window increased to $9 \times 9$.

The system then calculates the number of pixel components that form the set given in Eq. (18). This is essentially the number of pixel components under the temporary window that are within two estimated standard deviations of the noisy center pixel. 
Hore, Quu, and Wu: Noise estimation ...

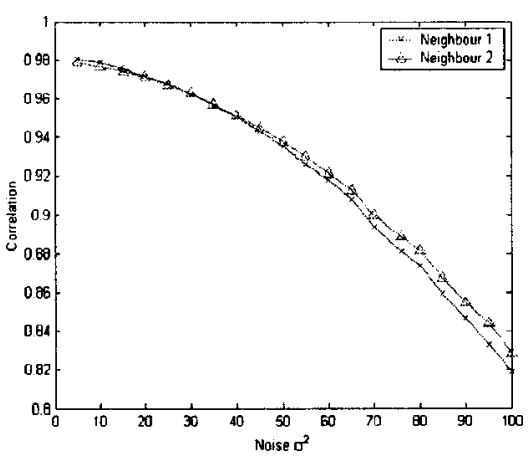

Fig. 2 Correlation between the original center pixel and its average two nearest neighbors.

$\mathbf{C}=\left\{x_{\epsilon} \mid x_{\epsilon} \in \mathbf{W}_{4},\left[x_{\epsilon(c p)}-2 \sigma_{\epsilon}\right] \leqslant x_{\epsilon} \leqslant\left[x_{\epsilon(c p)}+2 \sigma_{\epsilon}\right]\right\}$.

In Eq. (18), $\mathbf{C}$ is the contour set, $\mathbf{W}_{4}$ is the temporary window with increased dimensions, and $\sigma_{\epsilon}$ is the estimated noise standard deviation calculated from Eq. (20), which is described later in Sec. 2.3.

If we let $L_{c}$ denote the length of $\mathbf{C}$ and $L_{4}$ denote the size of the temporary window, then the value assigned to $v_{2}$ is given by the following expression:

$v_{2}=\left\{\begin{array}{ll}v_{2_{a}} & \text { if } L_{c}>\frac{1}{4}\left(L_{4}\right) \\ v_{2_{b}} & \text { if } L_{c} \leqslant \frac{1}{4}\left(L_{4}\right)\end{array}\right.$,

where again, $L_{c}$ denotes the number of pixel components that are within two standard deviations of the center pixel, and $v_{2_{a}}$ and $v_{2_{b}}$ are as per Eq. (17).

Through experimentation, we have found that on average, the two nearest-neighbor differences are highly correlated with the value of $\epsilon$. This is regardless of the noise type, although for additive/multiplicative noise the correlation is more pronounced. Figure 2 is a plot of the correlation between the average two nearest-neighbor distances and $\epsilon$. The correlation was calculated for increasing standard deviation in zero-mean Gaussian noise, averaged over a number of images with a variety of characteristics.

The third WS-filter variable is the average distance between the center pixel and its neighbors in the noisy input image that lie under the temporary filter window with increased dimensions. For additive and multiplicative noise, the approximate image may contain errors that are worse than those in the noisy input image. Note that this is not often the case. However, the use of the noisy input pixels rather than the approximate image pixels for this measure allows the weighted sum noise model to be more robust to such circumstances.

\subsection{Coefficient Partitioning}

The nonstationarity of image signals can often impair the performance of a restoration filter if it does not have the ability to adapt to changes in the image structure. In Refs. 23 and 24, a partitioning scheme was used to provide adaptive properties for linear filters. The partition-based linear
Table $1 \sigma_{\epsilon}^{2}$ operator.

\begin{tabular}{rrr}
\hline \hline 1 & -2 & 1 \\
-2 & 4 & -2 \\
1 & -2 & 1 \\
\hline
\end{tabular}

filters were shown to provide improved performance over local-invariant linear filters for the restoration of images corrupted with Gaussian noise. Partition techniques for adaptive filtering were also successfully applied to orderstatistics-based filters in the work by Chen and $\mathrm{Wu}$ with the partition-based median (PBM) filter. ${ }^{25}$

The filter proposed here also uses a partitioning scheme, where the partition indices, denoted as $p_{1}, p_{2}$, and $p_{3}$, are based on the local input signal characteristics. The coefficients within each partition are then optimized according to their specific image and noise characteristics. This provides greater robustness in the noise model and allows it to be optimized for variations in the noise and image structure.

The first partition index is a local measure of the noise variance and is calculated at each pixel location. The technique used to estimate the noise variance is the one proposed by Immerkaer. ${ }^{26}$ Although the algorithm was designed for additive Gaussian noise, the measure is used here only as an indexing parameter. Hence, it is only required to provide consistency in differentiating between changes in the noise characteristics.

The estimation algorithm uses a zero-mean and variance operator mask that is insensitive to image structure and has the form shown in Table 1.

When the operator is convolved with an image, the resulting pixels are equivalent to: $\left[4^{2}+4(-2)^{2}+4\right] \sigma_{\epsilon}^{2}$ $=36 \sigma_{\epsilon}^{2}$. Thus, the noise variance can be estimated for an entire image or a local region using the following equation:

$\sigma_{\epsilon}^{2}=\frac{1}{36(W-2)(H-2)} \sum_{s=2}^{W} \sum_{t=2}^{H}[I(s, t) * O]^{2}$,

where $[I(s, t) * O]$ denotes the convolution of the operator mask centered over the pixel at coordinates $s, t$ in the image. $W$ and $H$ are the width and height of the image, respectively.

To ensure that the number of partitions is kept to a reasonable level, the estimated noise variance is quantized to take on discrete values within a finite range. From experimentation, we have found that the optimal range is from 1 , where the noise variance is minimal, through to 6 , where the noise variance is severe. When the maximum value in the quantized range is increased above 6 , there is little or no improvement in the filter performance. This indicates that a quantization range of $1 \leqslant \sigma_{q}^{2} \leqslant 6$ provides adequate partitioning so that the noise characteristics between each level are dissimilar, while maintaining similar noise characteristics within each level.

The second partition index $p_{2}$ corresponds to the index of the center pixel component in $\tilde{X}_{c}$, the combined pixel set. This gives the index a range of $1 \leqslant p_{2} \leqslant L$, where $L$ is the length of the filter window. Due to the reduced aggregate ordering operation, when a center pixel component is 
placed at a high index, it is possible that it is either a highly corrupt pixel, or part of an edge or fine detail region in the underlying image structure. Similarly, when the center pixel component is placed at a low index in the ordered set, its aggregate interpixel distance is small, and hence, it is close to its neighboring pixels drawn from the approximate image.

In the case where the center pixel has a high index and may belong to a fine detailed segment, the WS-filter model needs to be able to recognize that a large value in $p_{1}$ is likely to be due to the neighboring pixels that do not belong to the center pixel segment. To achieve this, the off-line optimization of the coefficients assigned to a high $p_{2}$ partition index will generally result in a lesser weighting for $v_{1}$ than the coefficients assigned to a low $p_{2}$ partition.

The third partition index $p_{3}$ is used to measure the difference in spread of the pixel components from the ordered approximate and ordered combined sets. The spread is calculated as the difference between the components that have the smallest and largest aggregate interpixel distance, or equivalently the components at the first and last indices in the ordered sets. The difference in spread is then calculated using Eq. (21) and is quantized into five levels, as shown in Eq. (22).

$p_{3 q}=\left|\left(\tilde{x}_{c_{L}}-\tilde{x}_{c_{1}}\right)-\left(\tilde{x}_{a_{L}}-\tilde{x}_{a_{1}}\right)\right|$,

$p_{3 q}=\frac{p_{3 q}}{K} \times 5$.

In Eq. (22), $K$ is the maximum value that can result from Eq. (21). For spherical coordinates, the $\rho$ component will have a $K$ value that is the maximum magnitude of a 24-bit RGB pixel vector, i.e., 441.67. The $\theta$ and $\phi$ components will have a $K$ value of $K=\pi / 2$ rads, as this is the maximum angle between two RGB pixel vectors. If, on the other hand, the color space was 24-bit RGB, $K$ would equal 255 for all components. Similarly, for other color spaces, the value of $K$ depends on the dynamic range of the pixel vector component being processed.

Once the independent variables and the coefficient partition indices have been determined, the estimate of the noise present in the center pixel component is calculated using Eq. (14). The final output for the center pixel component is calculated by subtracting the result of Eq. (14), denoted as $\hat{\epsilon}$ from the noisy center pixel $x_{\epsilon(c p)}$, such that:

$Y=x_{\epsilon(c p)}-\hat{\epsilon}$.

\section{Experimental Results}

All of the images used in the experiments were 24-bit RGB images, with the WS-filter coefficients optimized using a set of more than 500 reference images. The training set was iterated over for each noise type (e.g., those presented in Sec. 1.2), where each noise model iteration included up to ten epochs per image. Thus, a total of 20,000 iterations were performed during the off-line optimization.

\subsection{Performance Measures}

The performance measures that have been employed are the peak signal-to-noise ratio (PSNR), measuring both the combined intensity and chromatic error as well as the chromatic error individually. The last performance measure is the normalized color difference (NCD). ${ }^{27}$

The PSNR was calculated for each color channel individually and then averaged for the three results. The following equation illustrates the PSNR calculation.

$\mathrm{PSNR}=10 \log _{10}\left(\frac{P_{\max }}{\mathrm{MSE}}\right)$.

The symbol $P_{\max }$ denotes the maximum component value, i.e., 255 for individual RGB components. MSE denotes the mean square error and is calculated using Eq. (25).

$\mathrm{MSE}=\frac{1}{W H} \sum_{s=1}^{W} \sum_{t=1}^{H}\left(\left|\mathbf{x}_{s, t}-\mathbf{y}_{s, t}\right|\right)^{2}$

In addition to the scalar PSNR measure, we introduce here a new PSNR calculation that measures the true (nonperceptual) error in chromaticity between two images. To achieve this, the proposed chromatic measure uses a slightly different MSE calculation, where the magnitude difference is changed to the angle between two RGB pixel vectors. This is illustrated in the following equation.

$\mathrm{MSE}_{\llcorner}=\frac{1}{W H} \sum_{s=1}^{W} \sum_{t=1}^{H}\left(\mathbf{x}_{s, t} \angle \mathbf{y}_{s, t}\right)^{2}$.

The symbol $\angle$ denotes the angle between the two vectors, calculated using the arc cosine of the inner product, such that:

$\mathbf{x}_{(s, t)} \angle \mathbf{y}_{(s, t)}=\cos ^{-1}\left[\frac{\mathbf{x}_{(s, t)} \cdot \mathbf{y}_{(s, t)}}{\left|\mathbf{x}_{(s, t)}\right|\left|\mathbf{y}_{(s, t)}\right|}\right]$.

Because the maximum angle between two RGB vectors will be $\pi / 2=1.5708$, the $P_{\max }$ from Eq. (24) will also be changed to $P_{\max }=1.5708$.

In addition to the chroma PSNR, the NCD is also used to quantify the color error between the filter output and the original image. Using a color space that has relative perceptual uniformity, such as $\mathrm{L}^{*} \mathrm{u}^{*} \mathrm{v}^{*}$ or $\mathrm{L}^{*} \mathrm{a}^{*} \mathrm{~b}^{*}$, the normalized Euclidean distance between the two pixels can provide an indication as to the perceived difference in color. Hence, the NCD provides an indication as to the perceived error in color. Equation (28) illustrates the NCD.

$\mathrm{NCD}=\frac{\sum_{s=1}^{W} \Sigma_{t=1}^{H}\left|\Delta L u v_{(s, t)}\right|}{\sum_{s=1}^{W} \Sigma_{t=1}^{H} \mid \operatorname{Luv}(s, t)}$.

$\Delta L u v$ is the Euclidean distance between the filter output pixel and the original image pixel at coordinates $(s, t)$ in $\mathrm{L}^{*} \mathrm{u}^{*} \mathrm{v}^{*}$ space. $L u v_{(s, t)}$ denotes the original image pixel in $\mathrm{L}^{*} \mathrm{u}^{*} \mathrm{v}^{*}$ space at the same coordinates. 
Table 2 Scalar median color space comparison.

\begin{tabular}{lccc}
\hline \hline & $\mid$ PSNR & $\angle$ PSNR & NCD $\left(10^{-2}\right)$ \\
\hline Spherical & 25.32 & 42.77 & 12.73 \\
RGB & 25.20 & 42.49 & 13.12 \\
YC $_{b} C_{r}$ & 25.29 & 42.58 & 12.91 \\
Luv & 24.88 & 42.36 & 12.95 \\
HSV & 20.77 & 38.04 & 23.78 \\
\hline \hline
\end{tabular}

\subsection{Filter Performance}

Table 2 shows the results of a multichannel scalar median filter applied to each channel individually for a number of different color spaces. The filters used in the experiment are a scalar median filter and the vector median filter, as proposed in Ref. 19. The image used was the Lena image corrupted by additive zero-mean Gaussian noise with a standard deviation of $\sigma=25$.

As the results from Tables 2 and 3 show, the scalar median operating on each individual pixel channel outperforms the vector median in all color spaces. Out of all the color spaces for either the scalar or vector median filter, the spherical and $\mathrm{YC}_{b} \mathrm{C}_{r}$ color spaces provide the best results.

This interesting result led to a second experiment where the aim was to compare the two color spaces for additive and multiplicative noise. The experiment was performed using more than 200 images. The average difference in performance between the two spaces was then calculated and is given in Table 4. The additive noise was $+\mathrm{UN}_{-40,40}$, while the multiplicative noise was $* \mathrm{UN}_{-40,40}$. The difference was calculated as the signed difference using the equation $D=R_{s p h}-R_{Y C b C r}$, where $R_{s p h}$ is the result for spherical coordinates and $R_{Y C b C r}$ is the result for the $\mathrm{YC}_{b} \mathrm{C}_{r}$ color space. Hence, a positive result occurs when the spherical coordinate space outperforms the $\mathrm{YC}_{b} \mathrm{C}_{r}$ space.

From these results, it can be seen that the two color spaces provide very similar results, with the spherical space performing only slightly better than the $\mathrm{YC}_{b} \mathrm{C}_{r}$ over the 200 image experiment.

In the results, the proposed filter is referred to as the $W S$-noise model ( $W S$-NM) filter. The other filters in the results are the partition-based median (PBM) filter, ${ }^{25}$ the partition-weighted sum (PWS) filter, ${ }^{23}$ and the fuzzy median filter (FMF). ${ }^{9}$

The intensity and color PSNR results for additive Gaussian noise are given in Tables 5 and 6, respectively, while the NCD results are shown in Table 7. As the results show,

Table 3 Vector median color space comparison.

\begin{tabular}{lccc}
\hline \hline & $|\mathrm{PSNR}|$ & $\angle \mathrm{PSNR}$ & $\mathrm{NCD}\left(10^{-2}\right)$ \\
\hline Spherical & 23.76 & 41.01 & 15.20 \\
$\mathrm{RGB}$ & 23.60 & 40.73 & 16.35 \\
$\mathrm{YC}_{b} \mathrm{C}_{r}$ & 23.72 & 40.88 & 15.99 \\
Luv & 22.26 & 41.56 & 14.88 \\
HSV & 20.43 & 36.44 & 25.86 \\
\hline \hline
\end{tabular}

Table 4 Spherical $/ \mathrm{YC}_{b} \mathrm{C}_{r}$ comparison.

\begin{tabular}{lccc}
\hline \hline & $|\mathrm{PSNR}|$ & $\angle \mathrm{PSNR}$ & $\mathrm{NCD}$ \\
\hline$+\mathrm{UN}_{-40,40}$ & 1.26 & 1.76 & 2.27 \\
$* \mathrm{UN}_{-40,40}$ & 2.05 & 2.12 & 2.23 \\
\hline \hline
\end{tabular}

Table 5 Intensity PSNR comparison for $+\mathrm{GN}_{0,25}$.

\begin{tabular}{lcccc}
\hline \hline & Lake & Mandrill & Lena & Cafe \\
\hline$W_{S}-\mathrm{NM}_{\text {sph }}$ & 28.08 & 24.04 & 27.34 & 27.98 \\
PBM $_{\text {rgb }}{ }^{25}$ & 26.89 & 21.40 & 26.52 & 26.38 \\
PWS $_{\text {rgb }}{ }^{23}$ & 26.29 & 21.63 & 25.99 & 26.64 \\
FMF $_{\text {rgb }}{ }^{9}$ & 26.21 & 20.05 & 25.23 & 25.90 \\
Unfiltered $^{20.62}$ & 20.35 & 20.39 & 20.31 \\
\hline \hline
\end{tabular}

Table 6 Color PSNR comparison for $+\mathrm{GN}_{0,25}$.

\begin{tabular}{|c|c|c|c|c|}
\hline & Lake & Mandrill & Lena & Cafe \\
\hline$W S-\mathrm{NM}_{\mathrm{sph}}$ & 40.02 & 41.25 & 45.16 & 43.79 \\
\hline $\mathrm{PBM}_{\mathrm{rgb}} 25$ & 35.59 & 39.62 & 43.35 & 40.97 \\
\hline $\mathrm{PWS}_{\mathrm{rgb}}{ }^{23}$ & 38.89 & 43.49 & 41.00 & 41.02 \\
\hline $\mathrm{FMF}_{\mathrm{rgb}}{ }^{9}$ & 35.50 & 38.22 & 43.03 & 40.95 \\
\hline Unfiltered & 30.58 & 35.32 & 36.54 & 33.16 \\
\hline
\end{tabular}

Table $7 \mathrm{NCD}\left(10^{-2}\right)$ comparison for $+\mathrm{GN}_{0,25}$.

\begin{tabular}{|c|c|c|c|c|}
\hline & Lake & Mandrill & Lena & Cafe \\
\hline$W S-\mathrm{NM}_{\mathrm{sph}}$ & 12.04 & 13.20 & 10.06 & 10.98 \\
\hline $\mathrm{PBM}_{\mathrm{rgb}} 25$ & 15.58 & 16.92 & 12.06 & 15.09 \\
\hline $\mathrm{PWS}_{\mathrm{rgb}} 23$ & 14.43 & 16.05 & 11.22 & 14.01 \\
\hline $\mathrm{FMF}_{\mathrm{rgb}} 9$ & 15.08 & 16.32 & 11.62 & 15.00 \\
\hline Unfiltered & 32.71 & 24.32 & 25.98 & 30.29 \\
\hline
\end{tabular}

Table 8 PSNR comparison for $+\mathrm{UN}_{20,20}$ noise.

\begin{tabular}{lcc}
\hline \hline & $|\mathrm{PSNR}|$ & $\angle \mathrm{PSNR}$ \\
\hline$W-\mathrm{NM}_{\text {sph }}$ & 32.03 & 42.61 \\
$\mathrm{PBM}_{\text {rgb }}{ }^{25}$ & 30.10 & 39.44 \\
PWS $_{\text {rgb }}{ }^{23}$ & 29.88 & 38.62 \\
$\mathrm{FMF}_{\text {rgb }}{ }^{9}$ & 28.92 & 39.05 \\
Unfiltered & 26.96 & 33.84 \\
\hline \hline
\end{tabular}


Table 9 PSNR comparison for $* \mathrm{UN}_{20,20}$ noise.

\begin{tabular}{lcc}
\hline \hline & $\mid$ PSNR $\mid$ & $\angle$ PSNR \\
\hline$W S-N_{\text {sph }}$ & 32.88 & 50.32 \\
PBM $_{\text {rgb }}{ }^{25}$ & 30.85 & 48.37 \\
PWS $_{\text {rgb }}{ }^{23}$ & 31.00 & 48.15 \\
FMF $_{\text {rgb }}{ }^{9}$ & 29.42 & 47.54 \\
Unfiltered $^{2}$ & 28.21 & 45.71 \\
\hline \hline
\end{tabular}

Table 10 NCD $\left(10^{-2}\right)$ comparison for $+\mathrm{UN}$ and $* U N$.

\begin{tabular}{lcc}
\hline \hline & $+\mathrm{UN}_{-20,20}$ & $* \mathrm{UN}_{-20,20}$ \\
\hline$W S-\mathrm{NM}_{\text {sph }}$ & 7.58 & 5.22 \\
$\mathrm{PBM}_{\text {rgb }}{ }^{25}$ & 9.98 & 6.41 \\
$\mathrm{PWS}_{\text {rgb }}{ }^{23}$ & 9.50 & 6.27 \\
$\mathrm{FMF}_{\text {rgb }}{ }^{9}$ & 10.06 & 6.75 \\
Unfiltered & 18.25 & 9.77 \\
\hline \hline
\end{tabular}

Table 11 Intensity PSNR comparison for $\mathrm{IM}_{30}$.

\begin{tabular}{lcccc}
\hline \hline & Lake & Mandrill & Lena & Cafe \\
\hline$W S-N M$ & 28.78 & 21.92 & 28.05 & 28.32 \\
PBM $_{\text {rgb }}{ }^{25}$ & 28.18 & 21.16 & 27.42 & 28.05 \\
PWS $_{\text {rgb }}{ }^{23}$ & 27.78 & 20.87 & 27.10 & 27.76 \\
FMF $_{\text {rgb }}{ }^{9}$ & 26.89 & 20.04 & 26.60 & 27.24 \\
Unfiltered & 15.22 & 16.51 & 15.66 & 14.84 \\
\hline \hline
\end{tabular}

Table 12 Color PSNR comparison for $\mathrm{IM}_{30}$.

\begin{tabular}{lcccc}
\hline \hline & Lake & Mandrill & Lena & Cafe \\
\hline$W S-$ NM $_{\text {sph }}$ & 45.19 & 42.76 & 46.26 & 45.22 \\
PBM $_{\text {rgb }}{ }^{25}$ & 44.47 & 40.94 & 46.05 & 44.52 \\
PWS $_{\text {rgb }}{ }^{23}$ & 43.98 & 40.85 & 45.09 & 44.12 \\
FMF $_{\text {rgb }}{ }^{9}$ & 40.97 & 40.59 & 45.99 & 43.55 \\
Unfiltered $^{2}$ & 30.54 & 32.09 & 31.81 & 31.50 \\
\hline \hline
\end{tabular}

Table $13 \mathrm{NCD}\left(10^{-2}\right)$ comparison for $\mathrm{IM}_{30}$.

\begin{tabular}{lrrrr}
\hline \hline & Lake & Mandrill & Lena & Cafe \\
\hline$W_{S}-\mathrm{NM}_{\text {sph }}$ & 3.64 & 10.08 & 4.78 & 4.83 \\
PBM $_{\text {rgb }}{ }^{25}$ & 5.76 & 12.31 & 6.30 & 6.29 \\
PWS $_{\text {rgb }}{ }^{23}$ & 5.24 & 13.04 & 6.07 & 6.14 \\
FMF $_{\text {rgb }}{ }^{9}$ & 5.83 & 13.76 & 6.32 & 6.76 \\
Unfiltered & 36.61 & 29.43 & 30.52 & 32.68 \\
\hline \hline
\end{tabular}

the proposed filter manages to outperform all of the other filters for the $+\mathrm{GN}_{0,25}$ noise model.

Tables 8 and 9 give the PSNR results for additive uniform and multiplicative uniform noise, respectively. Table 10 contains the NCD results for both additive and multiplicative uniform noise. Again, the results show the exceptional performance of the proposed filter over the others for both intensity and color preservation.

Although not originally designed for impulse noise restoration, a number of experiments were carried out to compare the performance of the proposed filter for impulse noise with the current state of the art filters. The noise density was $30 \%$ for each pixel component, with Tables 11 , 12, and 13 giving the intensity PSNR, color PSNR, and NCD results, respectively.

As was the case for additive and multiplicative noise, the proposed filter manages to outperform the others in terms of both intensity and color preservation.

\section{Conclusions}

A new color image restoration filter is proposed that employs a linear combination of a number of subfilter outputs and order statistics to estimate the amount of noise present in the center pixel. The weighting coefficients are partitioned according to the local signal content and estimated noise variance so that the filter is able to adapt to changes in the image and/or noise characteristics. The partitioned coefficients are optimized using a constrained recursive least mean square algorithm that in the past has shown improved results over using a nonconstrained, nonrecursive algorithm.

The filter operation is carried out as a scalar process in a color space that decouples the intensity and color information. After experimentation, it is found that the spherical coordinate representation of RGB pixel vectors provides improved results over many other color spaces for scalar processing. As the results of a number of experiments have shown, the proposed filter manages to outperform the current state of the art filter techniques in terms of both intensity and color preservation.

In addition to the proposed filter, a new color metric is introduced that provides a quantitative measure of the color difference between two images. The new measure can be applied using many of the existing error measures such as the mean square error, mean absolute error, and peak signal-to-noise ratio.

Possible future work may include performing a number of experiments with various robust estimators other than the linear combination approach to see if the results can be improved on. In addition to this, the partitioning scheme that provides the adaptive properties of the proposed filter may be further investigated.

\section{References}

1. J. Astola and P. Kuosmanen, Fundamentals of Nonlinear Digital Filtering, CRC Press, Boca Raton, FL (1997).

2. I. Pitas and A. N. Venetsanopoulos, "Order statistics in digital image processing," Proc. IEEE 80(12), 1893-1921 (1992).

3. P. S. Huber, Robust Statistics, Wiley, New York (1981).

4. F. R. Hampel, E. M. Ronchetti, P. J. Rousseeuw, and W. A. Stahel, Robust Statistics: The Approach Based on Influence Functions, Wiley, New York (1986).

5. J. W. Tukey, Exploratory Data Analysis, Addison-Wesley, Reading, MA (1977).

6. A. C. Bovik, T. Huang, and D. C. Munson, "A generalisation of 
Hore, Qiu, and Wu: Noise estimation ...

median filtering using linear combinations of order statistics," IEEE Trans. Acoust., Speech, Signal Process. ASSP-31(6), 1342-1350 (1983).

7. J. B. Bednar and T. L. Watt, "Alpha-trimmed means and their relationship to the median filters," IEEE Trans. Acoust., Speech, Signal Process. 32, 145-153 (Feb. 1984).

8. Y. H. Lee and S. A. Kassam, "Generalised median filtering and related nonlinear filtering techniques," IEEE Trans. Acoust., Speech, Signal Process. 33, 672-683 (June 1985).

9. K. Arakawa, "Median filters based on fuzzy rules and its application to image restoration," Fuzzy Sets Syst. 77, 3-13 (1996).

10. E. Abreu, M. Lightstone, S. K. Mitra, and K. Arakawa, "A new efficient approach for the removal of impulse noise from highly corrupted images," IEEE Trans. Image Process. 5(6), 1012-1025 (1996).

11. C. Kotropoulous and I. Pitas, "Constrained adaptive Ims 1-filters," Signal Process. 26, 335-358 (1992).

12. C. Kotropoulos and I. Pitas, "Adaptive 1ms 1-filters for noise suppression in images," IEEE Trans. Image Process. 5(12), 1596-1609 (1996).

13. T. Chen and H. R. Wu, "Recursive implementation of constrained lms 1-filters for image restoration," Signal Process. 81, 1101-1107 (May 2001)

14. K. Tang, J. Astola, and Y. Neuvo, "Multivariate order statistics in colour image processing," Proc. IEEE Intl. Conf. Systems Eng., pp. 584-587 (Sept. 1992).

15. P. E. Trahanias and A. N. Venetsanopoulos, "Vector directional filters-a new class of multichannel image processing filters," IEEE Trans. Image Process. 2(4), 528-534 (1993).

16. C. Kotropoulos and I. Pitas, "Multichannel l-filters based on marginal data ordering," IEEE Trans. Signal Process. 42(10), 2581-2595 (1994).

17. C. S. Regazzoni and A. Teschioni, "A new approach to vector median filtering based on space filling curves," IEEE Trans. Image Process. 6(7), 1025-1037 (1997)

18. M. Yu, G. Y. Jiang, D. M. Ha, T. Y. Choi, and Y. D. Kim, "New adaptive vector filter based on noise estimate," IEICE Trans. Fundamentals E86-A(6), 911-919 (1999).

19. J. Astola, P. Haavisto, and Y. Neuvo, "Vector median filter," Proc. IEEE 78(4), 678-689 (1990).

20. E. R. Davies, "Edge location shifts produced by median filters: theoretical bounds and experimental results," Signal Process. 16(2), 83-96 (1989).

21. E. R. Davies, "Median and mean filters produce similar shifts on curved boundaries," Electron. Lett. 27(10), 826-828 (1991).

22. J. D. Foley, A. van Dam, S. K. Feiner, and J. F. Hughes, Computer Graphics: Principles and Practics, 2nd ed., Addison-Wesley, Reading, MA (1995).

23. K. E. Barner, A. M. Sarhan, and R. C. Hardie, "Partition-based weighted sum filters for image restoration," IEEE Trans. Image Process. 8(5), 740-745 (1999).

24. A. M. Sarhan, R. C. Hardie, and K. E. Barner, "Partition-based adaptive estimation of single response evoked potentials," Proc. IEEE Conf. Acoustics, Speech Signal Process. 2, 961-964 (May 1995).

25. T. Chen and H. R. Wu, "Application of partition-based median filters for supressing noise in images," IEEE Trans. Image Process. 10(6), 829-836 (2001)

26. J. Immerkaer, "Note: Fast noise variance estimation," Comput. Vis Image Underst. 64(2), 300-302 (1996)

27. K. N. Plataniotis, D. Androutsos, and A. N. Venetsanopoulos, "Adaptive multichannel filters for colour image processing," Signal Process Image Commun. 11, 171-177 (1998).

Biographies and photographs of authors not available. 